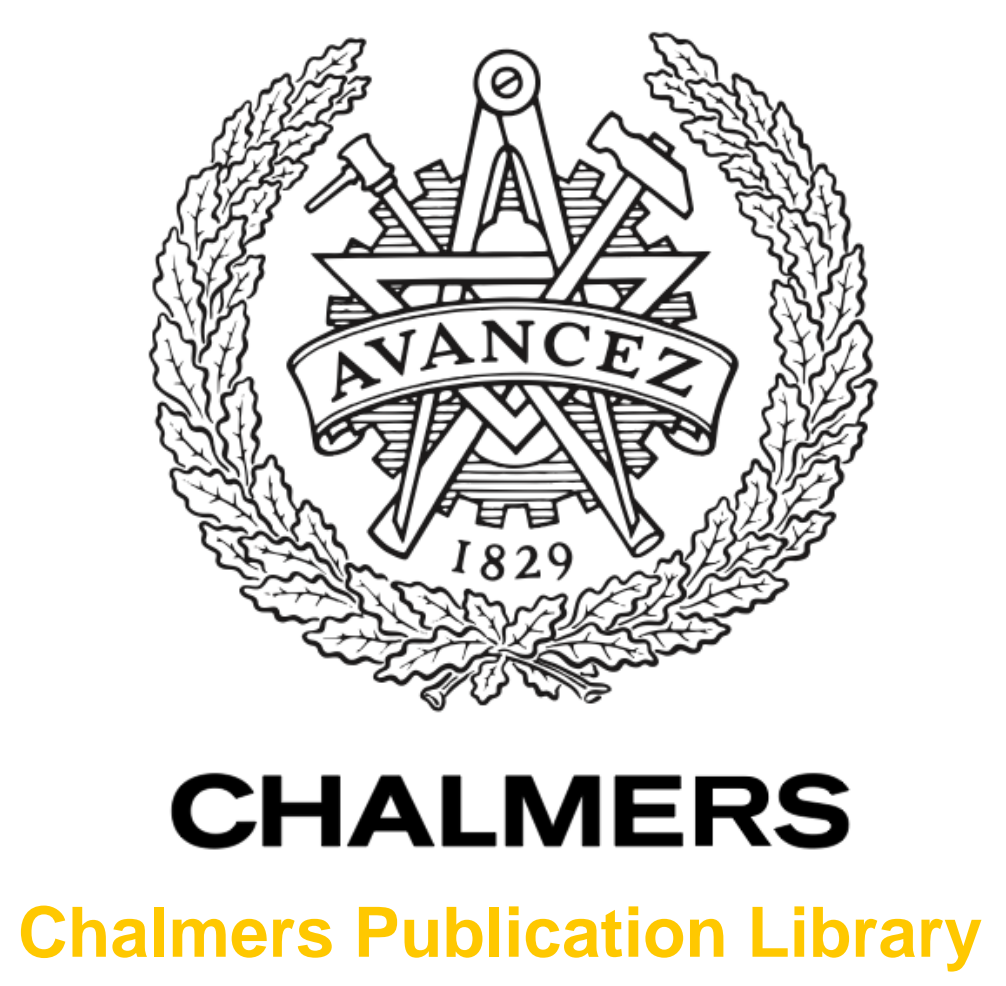

\title{
WiP abstract: Reception probability model for vehicular ad-hoc networks in the vicinity of intersections
}

This document has been downloaded from Chalmers Publication Library (CPL). It is the author's version of a work that was accepted for publication in:

2014 ACM/IEEE International Conference on Cyber-Physical Systems, ICCPS 2014

Citation for the published paper:

Steinmetz, E. ; Wildemeersch, M. ; Wymeersch, H. (2014) "WiP abstract: Reception probability model for vehicular ad-hoc networks in the vicinity of intersections". 2014 ACM/IEEE International Conference on Cyber-Physical Systems, ICCPS 2014 pp. 223.

http://dx.doi.org/10.1109/ICCPS.2014.6843736

Downloaded from: http://publications.lib.chalmers.se/publication/200864

Notice: Changes introduced as a result of publishing processes such as copy-editing and formatting may not be reflected in this document. For a definitive version of this work, please refer to the published source. Please note that access to the published version might require a subscription.

Chalmers Publication Library (CPL) offers the possibility of retrieving research publications produced at Chalmers University of Technology. It covers all types of publications: articles, dissertations, licentiate theses, masters theses, conference papers, reports etc. Since 2006 it is the official tool for Chalmers official publication statistics. To ensure that Chalmers research results are disseminated as widely as possible, an Open Access Policy has been adopted.

The CPL service is administrated and maintained by Chalmers Library. 


\section{WiP Abstract: Reception Probability Model for Vehicular Ad-Hoc Networks in the Vicinity of Intersections}

\author{
Erik Steinmetz \\ Dept. of Signals and Systems \\ Chalmers Univ. of Technology \\ Gothenburg, Sweden \\ estein@chalmers.se
}

\author{
Matthias Wildemeersch \\ Institute for Infocomm \\ Research \\ A*STAR, Singapore \\ stuwma@i2r.a-star.edu.sg
}

\author{
Henk Wymeersch \\ Dept. of Signals and Systems \\ Chalmers Univ. of Technology \\ Gothenburg, Sweden \\ henkw@chalmers.se
}

\begin{abstract}
In order to guide and validate the communication system design for vehicular ad-hoc networks (VANETs), and to obtain important insight about scalability and performance in these networks, analytical expressions of key performance metrics are necessary. In this study, we present an analytical model based on stochastic geometry to evaluate the reliability of packet transmission in VANETs in the vicinity of intersections.
\end{abstract}

\section{INTRODUCTION}

In VANETs, vehicles continuously share information with each other and their surrounding through wireless communication. This opens up for a new set of applications that are expected to enhance traffic safety and efficiency. The IEEE 802.11p standard has been defined to meet the communication demands of these applications. In order to guide and validate the communication system design, extensive simulations and measurements are often used to evaluate the reliability of packet transmission, e.g., for the important scenario of intersections [1]. We present an analytic performance assessment tool based on stochastic geometry, building on [2], that accounts for the spatial statistics of the vehicles for a road-crossing scenario.

\section{MAIN RESULT}

We consider an intersection scenario with four lanes (see Fig. 1), where each lane carries vehicles according to a one-dimensional Poisson point process with homogeneous intensity $\lambda_{i}, i \in\{1,2,3,4\}$. Neighbouring lanes are separated with a distance $d_{l}$. All vehicles broadcasts packets with a transmit power $P$ according to a slotted Aloha protocol with transmit probability $p \in[0,1]$. Furthermore, we consider a target receiver $(\mathrm{Rx})$ located a distance $d$ away from the intersection, and a target transmitter $(\mathrm{Tx})$ located a distance $r_{\mathrm{tx}}$ away from the receiver. The signal propagation model comprises Rayleigh fading, path loss $\left(A r_{\mathrm{tx}}\right)^{-\alpha}$ for antenna gain $A$ and path loss exponent $\alpha$, and white Gaussian noise with variance $\sigma^{2}$. Successful reception occurs when the signal-to-interference-plus-noise ratio (SINR) exceeds a threshold $\zeta$. The probability that Rx can de- code a packet from Tx is given by

$$
\begin{aligned}
& \mathbb{P}_{s}(\zeta)=\exp \left(-p \lambda_{1} \sqrt{\zeta} r_{\mathrm{tx}} \pi\right) \exp \left(-\frac{p \lambda_{2} \pi r_{\mathrm{tx}}^{2} \zeta}{\sqrt{d_{l}^{2}+r_{\mathrm{tx}}^{2} \zeta}}\right) \\
& \exp \left(-\frac{p \lambda_{3} \pi r_{\mathrm{tx}}^{2} \zeta}{\sqrt{\left(d-d_{l}\right)^{2}+r_{\mathrm{tx}}^{2} \zeta}}\right) \exp \left(-\frac{p \lambda_{4} \pi r_{\mathrm{tx}}^{2} \zeta}{\sqrt{d^{2}+r_{\mathrm{tx}}^{2} \zeta}}\right) \\
& \times \exp \left(\frac{-\zeta\left(A r_{\mathrm{tx}}\right)^{\alpha} \sigma^{2}}{P}\right),
\end{aligned}
$$

where the 5 factors correspond to the contributions from (i) the own lane of the Rx; (ii) the parallel lane; (iii) the near perpendicular lane; (iv) the far perpendicular lane; and (v) the noise and fading. The model provides fundamental insights in vehicular communication systems and can easily be extended to roads with different orientations and to scenarios where vehicles are clustered around the intersection. Ongoing work includes the adoption of realistic medium access control and shadowing.

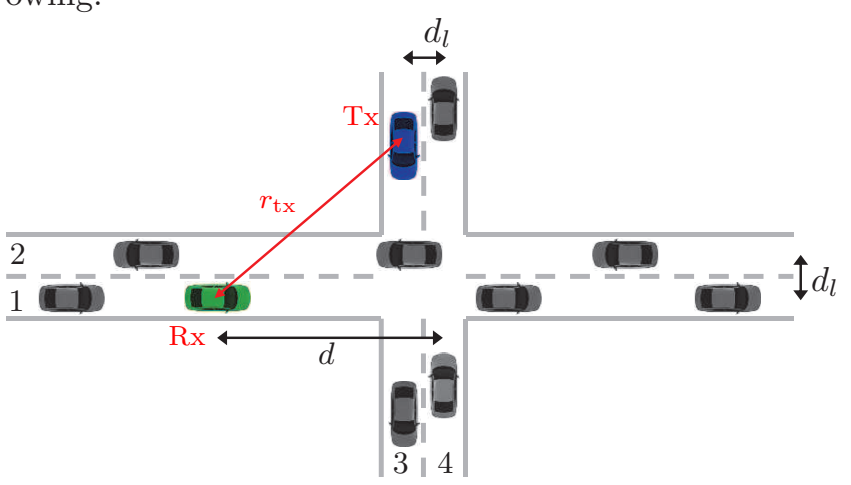

Figure 1: Intersection scenario with four lanes.

\section{REFERENCES}

[1] S. R. Azimi, G. Bhatia, and R. R. Rajkumar. Reliable intersection protocols using vehicular networks. ACM/IEEE 4th International Conference on Cyber-Physical Systems (ICCPS), 2013.

[2] B. Błaszczyszyn, P. Mühlethaler, and Y. Toor. Stochastic analysis of Aloha in vehicular ad hoc networks. Annals of telecommunications - Annales des télécommunications, 68(1-2):95-106, June 2012. 